\title{
Results of the ROTOR-program
}

\section{The long-term photometric variability of classical T Tauri stars ${ }^{\star}$}

\author{
K. N. Grankin ${ }^{1}$, S. Yu. Melnikov ${ }^{1}$, J. Bouvier ${ }^{2}$, W. Herbst ${ }^{3}$, and V. S. Shevchenko ${ }^{1, \star \star}$ \\ 1 Astronomical Institute of the Uzbek Academy of Sciences, Tashkent 700052, Uzbekistan \\ 2 Laboratoire d'Astrophysique, Observatoire de Grenoble, Université Joseph Fourier, BP 53, 38041 Grenoble Cedex 9, France \\ Astronomy Department, Wesleyan University, Middletown, CT 06459, USA
}

Received 24 April 2006 / Accepted 15 September 2006

\section{ABSTRACT}

\begin{abstract}
Context. T Tauri stars exhibit variability on all timescales, whose origin is still debated.
Aims. We investigate the long term variability of CTTs over up to 20 years, characterize it from a set of statistical parameters and discuss its origin.

Methods. We present a unique, homogeneous database of photometric measurements for Classical T Tauri stars extending up to 20 years. The database contains more than $21000 \mathrm{UBVR}$ observations of 72 CTTs. All the data were collected within the framework of the ROTOR-program at Mount Maidanak Observatory (Uzbekistan) and together they constitute the longest homogeneous, accurate record of TTS variability ever assembled. We characterize the long term photometric variations of 49 CTTs with sufficient data to allow a robust statistical analysis and propose an empirical classification scheme.

Results. Several patterns of long term photometric variability are identified. The most common pattern, exhibited by a group of 15 stars which includes $\mathrm{T}$ Tau itself, consists of low level variability $(\Delta V \leq 0.4 \mathrm{mag})$ with no significant changes occurring from season to season over many years. A related subgroup of 22 stars exhibits a similar stable long term variability pattern, though with larger amplitudes (up to $\Delta V \simeq 1.6 \mathrm{mag}$ ). Besides these representative groups, we identify three smaller groups of $3-5$ stars each which have distinctive photometric properties.

Conclusions. The long term variability of most CTTs is fairly stable and merely reflects shorter term variability due to cold and hot surface spots. Only a small fraction of CTTs undergo significant brightness changes on the long term (months, years), which probably arise from slowly varying circumstellar extinction.
\end{abstract}

Key words. stars: activity - stars: pre-main-sequence - stars: variables: general

\section{Introduction}

T Tauri stars (TTs) were first identified as a class of irregular variables by Joy $(1942,1945)$. The properties of these low mass pre-main sequence stars were reviewed by, e.g., Menard \& Bertout (1999) who emphasized two subgroups, the so-called "classical" T Tauri stars (CTTs) still actively accreting from their circumstellar disks and the "weak-line" T Tauri stars (WTTSs) no longer surrounded by a circumstellar disk. As discussed by Herbst et al. (1994), three types of day to week timescale variability can be identified in TTs. Type I variability, most often seen in WTTSs, is characterized by a low level periodic modulation of the stellar flux (a few $0.1 \mathrm{mag}$ ) and results from the rotation of a cool spotted photosphere. Type II variations have larger photometric amplitudes (up to 2 or 3 mag in extreme cases), are most often irregular but sometimes periodic, and are associated with short-lived accretion-related hot spots at the stellar surface of CTTs. Finally, the more rarely observed Type III variations are characterized by luminosity dips lasting from a few days up to several months, which presumably result from circumstellar dust obscuration, and are associated with the so-called UXOrs.

* $U B V R$ photometric catalogues are available in electronic form at the CDS via anonymous ftp to cdsarc.u-strasbg.fr (130.79.128.5) or via

http://cdsweb.u-strasbg.fr/cgi-bin/qcat?J/A+A/461/183

$\star \star$ Deceased March 2000.
The ROTOR program (Research Of Traces Of Rotation) is dedicated to the study of the photometric variability of pre-main-sequence (PMS) and main-sequence (MS) objects. The goal of the program is to obtain a long term homogeneous database of $U B V R$ observations of classical $\mathrm{T}$ Tauri stars (CTTs), weak-line T Tauri stars (WTTs), post-T Tauri stars (PTTs), Herbig AeBe stars (HAeBe), FU Orionis objects, and more evolved stars such as RS CVn, BY Dra, and FK Com stars. A detailed description of the program was given by Shevchenko (1989). More than 100000 observations of about 370 variable stars have been obtained since 1983 . Our list contains 72 CTTs, 45 WTTs, 54 PTTs, 88 HAeBe, and 11 FK Com stars. Observations were secured over more than 20 years by members of the Tashkent Astronomical Institute, including S.Yu. Melnikov (24\% of all observations), K. N. Grankin (23\%), M. Ibrahimov (14\%), S. D. Yakubov (10\%), O. V. Ezhkova (10\%), and V. Kondratiev (7\%). Preliminary results were published by Shevchenko et al. (1993b,a), Grankin et al. 1995; Shevchenko et al. 1998, Grankin (1997, 1998, 1999), Herbst \& Shevchenko (1999).

In this paper, we analyze the unique and extensive Mt. Maidanak database to investigate CTTs variability over a timescale of several years and, in some cases, several decades. In Sect. 2, we describe the stellar sample and the observations carried out for the last 20 years at Mt. Maidanak. In Sect. 3, we define the statistical parameters used to characterize the long 
Table 1. Results of Maidanak long-term photometry of CTTs.

\begin{tabular}{|c|c|c|c|c|c|c|c|c|}
\hline Name & $\mathrm{HBC}$ & $\mathrm{SpT}$ & $\mathrm{JD}_{\min }-\mathrm{JD}_{\max }$ & $N_{\mathrm{s}}$ & $V$ range & $N_{\text {obs }}$ & $\overline{B-V}$ & $\overline{V-R}$ \\
\hline AA Ori & 130 & $\mathrm{~K} 4$ & $47055-47777$ & 1 & $12.79-13.16$ & 11 & 1.28 & 1.23 \\
\hline AA Tau & 63 & K7 & $46690-53028$ & 15 & $12.30-14.14$ & 568 & 1.34 & 1.40 \\
\hline AS 205 & 254 & K5 & $46955-49588$ & 8 & $10.87-13.77$ & 362 & 1.24 & 1.55 \\
\hline AV Ori & 159 & K4 & $47040-47173$ & 1 & $12.84-14.42$ & 14 & 1.23 & 1.23 \\
\hline BD-22 4059 & 608 & G5 & $47670-49514$ & 5 & $10.10-10.28$ & 165 & 0.84 & 0.75 \\
\hline BM And & 318 & K5 & $47008-52908$ & 17 & $11.85-13.98$ & 999 & 1.13 & 1.07 \\
\hline BP Tau & 32 & K7 & $46679-52938$ & 11 & $11.67-12.99$ & 495 & 1.06 & 1.26 \\
\hline CI Tau & 61 & K7 & $47018-52935$ & 7 & $12.28-13.79$ & 319 & 1.37 & 1.55 \\
\hline CO Ori & 84 & F8 & $47031-52927$ & 16 & $9.81-12.73$ & 541 & 1.06 & 0.99 \\
\hline CX Tau & 27 & M2.5 & $51782-51841$ & 1 & $13.62-13.80$ & 15 & 1.51 & 1.65 \\
\hline CY Tau & 28 & M1 & $52579-53028$ & 1 & $12.94-13.59$ & 19 & 1.07 & 1.53 \\
\hline DD Tau & 30 & M1 & $48123-49275$ & 4 & $13.45-15.15$ & 114 & 1.07 & 1.76 \\
\hline DE Tau & 33 & M2 & $46681-47382$ & 1 & $12.82-13.95$ & 23 & 1.43 & 1.64 \\
\hline DF Tau & 36 & M0 & $45920-53028$ & 19 & $10.64-12.74$ & 929 & 1.10 & 1.45 \\
\hline DG Tau & 37 & M & $45928-52938$ & 18 & $11.23-13.58$ & 710 & 1.06 & 1.35 \\
\hline DH Tau & 38 & M1e & $52932-52938$ & 1 & $13.20-13.73$ & 5 & 0.89 & 1.36 \\
\hline DI Cep & 315 & G8 & $46966-52907$ & 17 & $11.14-11.67$ & 1231 & 0.89 & 0.88 \\
\hline DI Tau & 39 & M0 & $46710-51841$ & 11 & $12.69-12.97$ & 435 & 1.59 & 1.50 \\
\hline DK Tau & 45 & K7 & $47022-52937$ & 11 & $11.54-14.46$ & 429 & 1.27 & 1.43 \\
\hline DL Tau & 58 & K7 & $47017-52935$ & 8 & $12.39-13.89$ & 370 & 1.09 & 1.42 \\
\hline DM Tau & 62 & M1 & $47028-52935$ & 6 & $13.43-14.32$ & 162 & 1.07 & 1.51 \\
\hline DN Tau & 65 & M0 & $46681-52914$ & 10 & $12.08-12.54$ & 369 & 1.33 & 1.35 \\
\hline DR Tau & 74 & K5 & $46686-52924$ & 16 & $10.76-12.78$ & 642 & 0.82 & 1.05 \\
\hline DS Tau & 75 & K5 & $46680-48230$ & 4 & $11.58-12.68$ & 199 & 0.91 & 1.09 \\
\hline EH Cep & 307 & $\mathrm{~K} 2$ & $46970-52907$ & 17 & $11.52-13.44$ & 1296 & 1.19 & 1.03 \\
\hline EZ Ori & 114 & G0 & $47048-53022$ & 7 & $11.48-12.25$ & 222 & 0.84 & 0.77 \\
\hline GG Tau & 54 & K7 & $46686-49287$ & 7 & $11.91-12.48$ & 345 & 1.42 & 1.44 \\
\hline GI Tau & 56 & K6 & $46681-52935$ & 8 & $12.30-14.85$ & 344 & 1.46 & 1.57 \\
\hline GK Tau & 57 & K7 & $46682-52935$ & 8 & $11.94-13.58$ & 376 & 1.43 & 1.44 \\
\hline GM Aur & 77 & K3 & $46681-50046$ & 9 & $11.74-12.35$ & 349 & 1.21 & 1.22 \\
\hline GW Ori & 85 & G5 & $47031-52927$ & 15 & $9.74-10.53$ & 531 & 0.99 & 0.94 \\
\hline GX Ori & 89 & $\mathrm{~K} 1$ & $47031-47169$ & 1 & $13.11-13.47$ & 20 & 0.95 & 1.02 \\
\hline Haro 1-1 & 256 & K5.7 & $46964-49190$ & 6 & $12.80-13.82$ & 166 & 1.27 & 1.45 \\
\hline Haro 1-14 & 267 & M0 & $46964-50660$ & 6 & $13.75-15.03$ & 154 & 1.52 & 1.68 \\
\hline Haro 1-4 & 257 & K6.7 & $46964-48837$ & 5 & $13.29-13.81$ & 131 & 1.75 & 1.85 \\
\hline Haro 1-8 & 261 & K5 & $46965-49190$ & 4 & $13.74-14.22$ & 133 & 1.69 & 1.70 \\
\hline Haro1-14c & 644 & K3 & $46607-46649$ & 1 & $12.13-13.13$ & 27 & 1.67 & \\
\hline HI Ori & 93 & K4 & $47030-52176$ & 7 & $12.63-13.86$ & 214 & 1.28 & 1.20 \\
\hline HL Tau & 49 & K7-M2? & $48147-48954$ & 2 & $14.22-14.63$ & 48 & 1.42 & 1.54 \\
\hline HN Tau & 60 & K5 & $47028-49277$ & 6 & $12.63-14.94$ & 163 & 0.97 & 1.30 \\
\hline HP Tau & 66 & K3 & $46702-47421$ & 1 & $13.35-14.20$ & 35 & 1.78 & 1.69 \\
\hline LkCa 8 & 385 & M0 & $48953-52935$ & 1 & $12.83-13.44$ & 26 & 1.31 & 1.39 \\
\hline LkCa 15 & 419 & K5 & $48859-50760$ & 5 & $11.85-13.34$ & 123 & 1.26 & 1.16 \\
\hline LkHa 172 & 298 & K4 & $48087-49962$ & 2 & $14.58-14.98$ & 64 & 1.44 & 1.36 \\
\hline LkHa 191 & 301 & K0 & $46618-49956$ & 5 & $12.87-13.09$ & 326 & 1.10 & 1.04 \\
\hline LkHa 200 & 6 & K1 & $46258-46802$ & 1 & $13.51-13.68$ & 29 & 1.23 & 1.10 \\
\hline LkHa 209 & 194 & G8 & $46722-50044$ & 8 & $11.64-12.06$ & 193 & 0.83 & 0.80 \\
\hline LkHa 228 & 295 & K1.2 & $46261-50017$ & 2 & $12.87-13.06$ & 48 & 0.84 & 0.78 \\
\hline LkHa 274 & 203 & K4 & $46722-50024$ & 3 & $13.16-13.49$ & 92 & 1.26 & 1.20 \\
\hline LkHa 321 & 303 & $\mathrm{G}$ & $46985-50020$ & 7 & $12.25-12.44$ & 547 & 1.23 & 1.17 \\
\hline LX Ori & 133 & K3 & $47039-51834$ & 6 & $11.98-12.34$ & 191 & 1.10 & 1.00 \\
\hline Oph 6 & 653 & $\mathrm{~K}$ & $48419-49245$ & 1 & $13.18-13.45$ & 48 & 1.69 & 1.75 \\
\hline RW AurAB & 80.81 & $\mathrm{~K} 1-\mathrm{K} 3$ & $46680-52924$ & 16 & $9.32-11.75$ & 534 & 0.72 & 0.87 \\
\hline RY Tau & 34 & $\mathrm{~K} 1$ & $45968-53022$ & 19 & $9.55-11.56$ & 885 & 1.03 & 1.07 \\
\hline $\mathrm{S} \mathrm{CrA}$ & 286 & K6 & $47673-48838$ & 3 & $10.40-12.32$ & 122 & 1.00 & 1.18 \\
\hline SU Aur & 79 & $\mathrm{G} 2$ & $45676-52926$ & 19 & $8.92-10.02$ & 793 & 0.92 & 0.83 \\
\hline SW Ori & 115 & K4 & $47041-50022$ & 8 & $12.42-13.69$ & 153 & 1.04 & 0.97 \\
\hline T Tau & 35 & K0 & $46665-52937$ & 17 & $9.75-10.18$ & 982 & 1.17 & 1.13 \\
\hline UX TauAB & 42.43 & M1-K2 & $47378-49287$ & 6 & $10.64-12.77$ & 318 & 1.17 & 1.06 \\
\hline UY Aur & 76 & K7 & $46681-52914$ & 14 & $11.28-13.68$ & 427 & 1.27 & 1.43 \\
\hline UZ Tau & 52 & M1.3 & $46383-47101$ & 1 & $12.25-13.11$ & 15 & 1.19 & 1.66 \\
\hline V1082 Cyg & 728 & & $48059-50022$ & 6 & $12.62-13.67$ & 461 & 0.76 & 1.00 \\
\hline V1121 Oph & 270 & K5 & $46955-52876$ & 14 & $10.80-11.83$ & 601 & 1.28 & 1.36 \\
\hline V2058 Oph & 259 & K6 & $46607-49194$ & 7 & $12.60-13.09$ & 209 & 1.49 & 1.60 \\
\hline V2062 Oph & 268 & K2 & $46607-49194$ & 7 & $12.20-13.05$ & 223 & 1.34 & 1.39 \\
\hline V360 Ori & 144 & K6 & $47060-50022$ & 5 & $12.40-12.71$ & 159 & 1.39 & 1.30 \\
\hline
\end{tabular}


Table 1. continued.

\begin{tabular}{lrllrlrll}
\hline \hline Name & HBC & SpT & $\mathrm{JD}_{\min }-\mathrm{JD}_{\max }$ & $N_{\mathrm{s}}$ & $V$ range & $N_{\text {obs }}$ & $\overline{B-V}$ & $\overline{V-R}$ \\
\hline V521 Cyg & 299 & G8 & $46609-52899$ & 17 & $13.37-14.70$ & 1140 & 1.27 & 1.20 \\
V625 Ori & 183 & K6 & $47061-50010$ & 5 & $12.61-13.97$ & 114 & 1.08 & 1.15 \\
V649 Ori & 86 & G8 & $47031-52176$ & 7 & $11.87-12.31$ & 275 & 1.12 & 1.09 \\
V853 Oph & 266 & M1.5 & $46607-51014$ & 7 & $12.61-13.87$ & 238 & 1.10 & 1.63 \\
XZ Tau & 50 & M3 & $48142-48954$ & 3 & $12.96-15.17$ & 74 & 1.40 & 1.88 \\
YY Ori & 119 & K5.M0 & $47041-47170$ & 1 & $13.20-14.08$ & 16 & 0.77 & 1.01 \\
\hline
\end{tabular}

term variability of CTTs. In Sect. 4, we provide the results of the statistical analysis of CTTs light curves and offer in Sect. 5 an empirical classification of their long term photometric patterns. In Sect. 6, we attempt to relate the various types of photometric patterns seen to the underlying causes of variability.

\section{Star sample and observations}

All $U B V R$ data were obtained at Mount Maidanak Observatory (longitude: $\mathrm{E} 4^{\mathrm{h}} 27^{\mathrm{m}} 35^{\mathrm{s}}$; latitude: $+38^{\circ} 41^{\prime}$; altitude: $2540 \mathrm{~m}$ ) in Uzbekistan. Results of astroclimate studies at Maidanak observatory have been summarized by Ehgamberdiev et al. (2000), who show that it is among the best observatories in the world, in many respects. We targeted 72 CTTs for long-term monitoring from the list of Herbig \& Bell (1988). More than $21000 U B V R$ magnitudes were collected for these objects from 1983 up to 2003, although the number of $U$ magnitudes is relatively small compared to the other colors due to the faintness of the stars at those wavelengths. All the observations were obtained at two telescopes ( 0.6 and $0.48 \mathrm{~m}$ reflectors) using a single-channel pulse-counting photometer with photomultiplier tubes. As a rule, each program star was measured once per night at minimal airmass. We secured up to $120(U) B V R$ measurements for each sample target during each observational season lasting several months. The number of measurements depends on the object's visibility from Mt. Maidanak. The largest number of measurements was thus obtained for CTTs in Cygnus, Cassiopea, and Taurus, and fewer in Ophiuchus and Orion. magnitudes are provided in the Johnson $U B V R$ system. The rms error of a single measurement in the instrumental system for a star brighter than $12^{\mathrm{m}}$ in $V$ is about 0.01 in $B V R$ and 0.05 in $U$.

Observations were carried out either differentially using a nearby reference star (see Strauzis 1977) or directly by estimating the nightly extinction (Nikonov 1976). In the latter case, several reference stars were observed every night to derive the extinction coefficients in each filter. Selected standard stars (Landolt 1983, 1992) were observed and used to calibrate instrumental magnitudes on the Cousins system. We then transformed the magnitudes to the Johnson $U B V R$ system using the relationship from Landolt $(1983):(V-R)_{\mathrm{c}}=-0.0320+0.71652 \times$ $(V-R)_{J}$. The formal accuracy of this reduction step is 0.01 . All observed local times have been converted to heliocentric julian days (JDH). A detailed description of the equipment, observation techniques, and data processing is given in Shevchenko (1989).

The results of the Maidanak program of homogeneous longterm photometry of CTTs are summarized in Table 1. Columns are: star's name, star's number in Herbig \& Bell (1988)'s catalogue, spectral type, time span of observations in JD, number of seasons observed, photometric range in the $V$ band, number of observations in the $V$ band, and average values of $B-V$ and $V-R$ colors. The complete Maidanak database for CTTs is available in electronic form at the CDS, Strasbourg.

\section{Statistical parameters}

We define here the statistical parameters which allow us to characterize the long term photometric variability of CTTs. To increase the reliability of the statistical analysis we consider only observing seasons having at least 12 measurements and further exclude those stars which have less than 5 seasons of observations. As a result we retain 49 CTTs for the analysis. We also performed a more stringent analysis by retaining only 39 stars having more than 20 measurements per season and more than 5 seasons of observations. The results of the 2 analyzes are not significantly different from one another. We thus conclude that our selection criteria for the first sample of 49 stars provide robust statistical parameters (mean, standard deviations, minimum and maximum levels, etc.).

For each object, we compute the mean light level $\left(V_{\mathrm{m}}{ }^{(i)}\right)$ in season $(i)$ and the corresponding photometric range $\left(\Delta V^{(i)}\right)$ for that season. Then we average the $\left(V_{\mathrm{m}}{ }^{(i)}\right)$ over all seasons to yield $\left(\overline{V_{\mathrm{m}}}\right)$ and also compute its standard deviation $\left(\sigma_{V_{\mathrm{m}}}\right)$ based on the scatter of the seasonal means. Similarly, we compute the average $V$ band photometric amplitude over all seasons $\left(\overline{\Delta V}=\frac{\sum \Delta V^{(i)}}{N_{\mathrm{s}}}\right.$, where $N_{\mathrm{s}}$ is the number of observational seasons) and its standard deviation $\left(\sigma_{\Delta V}\right)$. We also use the ratio $\frac{\sigma_{\Delta V}}{\overline{\Delta V}}$.

In addition to statistical parameters, we investigate the color behavior of CTTs. Previous studies of the short term variability of CTTs have shown that most stars tend to redden in color indices (such as $V-R$ and $B-V$ ) when fading (e.g. Herbst et al. 1994). This behavior can result from surface spots, either cold or hot (cf. Bouvier et al. 1995), or from variable extinction by circumstellar dust. We characterize the color behavior of CTTs by computing the color slopes $\frac{\Delta(B-V)}{\Delta V}$ and $\frac{\Delta(V-R)}{\Delta V}$ from least-square fits, and the correlation coefficients $\rho_{B-V}$ and $\rho_{V-R}$. We used the full set of data pertaining to each object in order to compute these parameters since there are usually too few measurements per season to obtain accurate seasonal color slopes. For most stars, a strong correlation is found between color and brightness variations with little evidence for significant variations from season to season (cf., e.g., Fig. 8).

The resulting values of statistical and color parameters for 49 CTTs are listed in Table 2. The columns in Table 2 provide the star's name, the number of seasons $N_{s}^{*}$ with at least 12 measurements, the mean brightness level $\left(\overline{V_{\mathrm{m}}}\right)$ and its seasonal standard deviation $\left(\sigma_{V_{\mathrm{m}}}\right)$, the average photometric range $(\overline{\Delta V}$, averaged over all seasons) and its seasonal standard deviation $\left(\sigma_{\Delta V}\right)$, the fractional variation of photometric amplitude as measured by the ratio $\frac{\sigma_{\Delta V}}{\overline{\Delta V}}$, and the color slopes $\frac{\Delta(B-V)}{\Delta V}$ and $\frac{\Delta(V-R)}{\Delta V}$ together with their correlation coefficients $\rho_{B-V}$ and $\rho_{V-R}$. Two additional parameters discussed below (cf. Sect. 4) are also listed, which characterize the relative seasonal variability of the maximum and minimum light levels $\left(C 2=\sigma_{V_{\min }} / \sigma_{V_{\max }}\right)$ and the preferred brightness state of the object on the long term $\left(C 1=\left\langle\frac{V_{\operatorname{med}}-V_{\min }}{\Delta V}\right\rangle\right.$ where $V_{\text {med }}$ is the median light level of the object in a given 
Table 2. Statistical properties of CTTs light curves (see text).

\begin{tabular}{|c|c|c|c|c|c|c|c|c|c|c|c|c|}
\hline Star Name & $N_{\mathrm{s}}^{*}$ & $\overline{V_{\mathrm{m}}}$ & $\sigma_{V_{\mathrm{m}}}$ & $\overline{\Delta V}$ & $\sigma_{\Delta V}$ & $\frac{\sigma_{\Delta V}}{\overline{\Delta V}}$ & $\frac{\Delta(B-V)}{\Delta V}$ & $\rho_{B-V}$ & $\frac{\Delta(V-R)}{\Delta V}$ & $\rho_{V-R}$ & $c 1$ & 2 \\
\hline AA Tau & 15 & 12.917 & 0.143 & 1.133 & 0.322 & 0.285 & 0.017 & 0.04 & 0.093 & 0.54 & 0.423 & 0.304 \\
\hline AS 205 & 8 & 12.259 & 224 & 2.002 & 59 & 0.229 & .129 & .51 & 0.239 & 89 & .997 & 452 \\
\hline BD-224059 & 5 & 10.169 & 6 & 6 & & 0 & 73 & & 483 & 53 & & 427 \\
\hline & 17 & 12 & & & & & 35 & & 77 & & & 442 \\
\hline BP & 11 & & & & & & & & 3 & & & 499 \\
\hline CI Tau & 7 & 107 & & 74 & & & 340 & & 300 & & & 532 \\
\hline CO Ori & 15 & 960 & 22 & 73 & & 261 & 061 & 66 & 105 & & 668 & 425 \\
\hline DF Tau & 19 & 983 & 95 & & 92 & 217 & .392 & 83 & 386 & 92 & 42 & 629 \\
\hline DG Tau & 18 & 207 & .541 & 723 & 0.247 & 341 & .144 & .74 & 187 & & 32 & .484 \\
\hline DI Cep & 17 & 11.389 & 0.059 & 280 & 0.059 & 211 & .190 & 0.46 & .178 & o & 73 & .561 \\
\hline DI Tau & 9 & 12.843 & 0.013 & 156 & 0.064 & .409 & 0.147 & 0.09 & 0.440 & 7 & .998 & 0.428 \\
\hline DK Tau & 11 & .583 & .178 & 863 & 0.590 & 316 & 0.151 & 0.58 & 0.223 & 0.90 & .250 & 0.444 \\
\hline DL Tau & 9 & 146 & 54 & 015 & & 238 & 0.189 & 0.61 & 0.300 & 85 & .176 & .531 \\
\hline DM Tau & 6 & 928 & 93 & .591 & 54 & 091 & .596 & 0.79 & 0.472 & 83 & 285 & 0.571 \\
\hline DN Tau & 10 & 12.332 & & 289 & & 0.279 & 0.447 & 0.46 & 0.223 & 4 & & .542 \\
\hline & 16 & 11.660 & & 1.271 & & 0.292 & 0.173 & & 0.229 & & & 432 \\
\hline & 17 & 12.391 & & 1.064 & & 0.303 & 0.046 & & 95 & & & 423 \\
\hline & 7 & & & 4 & & & 56 & & 36 & & & 350 \\
\hline & 7 & & & & & & & & & & & 538 \\
\hline & 8 & & & & & & & & & & & 377 \\
\hline & 8 & & & & & & & & & & & 382 \\
\hline & 9 & 052 & & & & & & & & & & 500 \\
\hline & 15 & 8 & & 0 & & 1 & 0.159 & & 0 & & & .360 \\
\hline $\mathrm{H}$ & 6 & 355 & 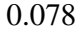 & 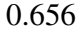 & & 8 & 0.390 & & 4 & & & 520 \\
\hline & 6 & 20 & & 3 & & 3 & 0.0 & $0 .($ & 66 & & & 0.433 \\
\hline & 5 & 529 & & 1 & & & 366 & & 4 & & & .609 \\
\hline & 7 & 347 & & 8 & & & & & & & & .469 \\
\hline & 6 & 93 & & 6 & & & 5 & & & & & 612 \\
\hline & 5 & -4 & & & & & & & & & & 263 \\
\hline & 5 & & & & & & & & & & & 421 \\
\hline & 8 & & & & & & & & & & & 435 \\
\hline & 7 & & & & & & & & & & & 492 \\
\hline & 6 & & & & & & & & & & & 466 \\
\hline & 16 & & & & & & & & & & & 488 \\
\hline & 11 & & & & & & & & & & & 462 \\
\hline & 1 & & & & & & & & & & & 354 \\
\hline & 5 & & & & & & & & & & & 26 \\
\hline & 17 & 72 & & & & & 40 & & 03 & & & 0.541 \\
\hline & 6 & 286 & & & & 3 & 0.2 & & 0.246 & & & 0.342 \\
\hline & 13 & 560 & & 0 & & & 0. & & 14 & & & .544 \\
\hline & 6 & 42 & & 8 & & 4 & 56 & & 0.222 & & & .552 \\
\hline & 14 & 30 & & & & 2 & 7 & & 4 & & & .569 \\
\hline $\mathrm{V}_{2}^{2}$ & 7 & 25 & & 7 & & 9 & 0. & & 42 & & & 544 \\
\hline $\mathrm{V} 2$ & 7 & 176 & & 4 & & 3 & 33 & & 41 & & & 483 \\
\hline & 5 & 529 & & & & & 0.311 & & 0.149 & 39 & & 0.496 \\
\hline Cyg & 17 & .736 & & 0.732 & & 0.397 & 0.275 & & 0.246 & 71 & 91 & 0.289 \\
\hline Ori & 5 & 136 & & 0.862 & & 0.474 & 0.385 & & 0.258 & & 64 & .458 \\
\hline V64 & 7 & 12.078 & & 0.262 & & 0.176 & 0.249 & & 0.238 & & 71 & 0.504 \\
\hline V853 Oph & 7 & 13.443 & 0.142 & 0.580 & 0.203 & 0.350 & 0.533 & 0.61 & 0.544 & 0.83 & 2.111 & 0.596 \\
\hline
\end{tabular}

season and the bracket indicates an average over all seasons). For instance, a low $C 1$ value indicates an object which spends more time close to its maximum brightness level.

In order to illustrate the meaning of these parameters, an example of the statistical analysis is shown in Fig. 1 for UY Aur. The long-term $V$-band light curve obtained at Maidanak Observatory is shown in the upper panel. In the lower panel, some of the statistical parameters are illustrated. The mean light level for each season of observations $\left(V_{m}{ }^{(i)}\right)$ is marked by a filled circle and the minimum and maximum brightness by crosses. Statistical parameters are not computed for two seasons (2001 and 2004) which contain less than 12 measurements. The solid line joins values of average brightness and demonstrates significant changes on years timescale. The photometric amplitude is also seen to vary on the long term.

Figures $2 \mathrm{a}$ and $2 \mathrm{~b}$ display the light curves of the other 48 CTTs on which we applied the same statistical analysis. It is immediately obvious that different photometric patterns occur on the long term. We discuss these patterns with the help of the above defined statistical parameters in the next section.

\section{Results}

In this section, we present the results of the statistical analysis of the long term light curves. The histogram of the average $V$-band amplitude of variability for the CTTs in our sample is shown in 

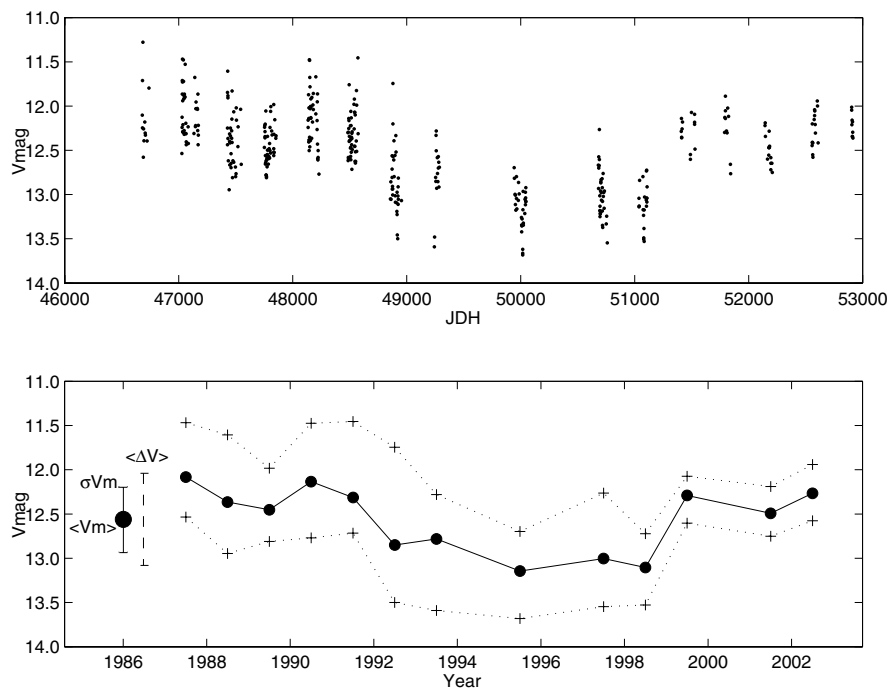

Fig. 1. The long term $V$-band light curve of the CTTs UY Aur. Top panel: photometric measurements; lower panel: statistical analysis (see text for details).

Fig. 3. The distribution is rather large, with $\overline{\Delta V}$ ranging from 0 . 1 up to $2^{\mathrm{m}}$.

Nearly half of the CTTs (21) exhibit small ranges of variability on the long term, from 0.1 to 0.4 . A roughly equal number (24) display larger ranges, from $0^{\mathrm{m}} \cdot 6$ to 1.5 in the $V$-band. Only a few CTTs (4) exhibit average $V$-band ranges larger than 1 . 5 and up to $2^{\mathrm{m}}$ over the years. These are BM And, GI Tau, AS 205, and DK Tau.

A histogram showing the frequency distribution of the measured variations of mean light level from season to season of the 49 CTTs in our statistical sample is displayed in Fig. 4. Specifically, this variation is measured by the standard deviation of the seasonal mean light levels $\left(\sigma_{V_{\mathrm{m}}}\right)$. The histogram is strongly peaked, with the large majority of CTTs demonstrating little variation in their mean light level over the years $\left(\sigma_{V_{\mathrm{m}}} \simeq 0\right.$. $\left.1-0.2\right)$. However, a few CTTs (6) are characterized by significant changes in average brightness, with $\sigma_{V_{\mathrm{m}}}$ ranging from 0.25 to 0.65 (cf. Fig. 6). These are CO Ori, DG Tau, HN Tau, UY Aur, RY Tau, and HI Ori. Figures 1 and 2a show that the mean light level can vary smoothly on a timescale of several years (e.g. UY Aur) or, on the contrary, can change quite abruptly from one season to the next (e.g. DG Tau).

Similarily, the variations of the $V$-band range from season to season are small for most CTTs, amounting to only a few tenths of a magnitude at most, as shown by the histogram of $\sigma_{\Delta V}$ in Fig. 5. We caution that in seasons where the number of data is not large or in the case of stars where the variations are rapid and short-lived, we may be underestimating the true photometric range. Nonetheless, it appears that this histogram is not as peaked towards small values as is the histogram of $\sigma_{V_{\mathrm{m}}}$ (Fig. 4) but displays a more continuous distribution. We find that the relative changes in amplitude $\left(\frac{\sigma_{\Delta V}}{\overline{\Delta V}}\right)$ are usually constrained to lie between 0.1 and 0.5 , except for 4 CTTs which do exhibit larger seasonal variations up to 0.9 (EZ Ori, SU Aur, V2062 Oph, and GW Ori, see Fig. 6). In other words, CTTs with the largest photometric amplitudes are also those whose amplitude varies the most on a timescale of years.

That most CTTs exhibit relatively similar variations in mean brightness level and fractional amplitudes is best seen in Fig. 6 where the 2 quantities are plotted against each other. The vast majority of CTTs in our sample lie in a small region of the diagram delimited by $\sigma_{V_{\mathrm{m}}} \leq 0.3$ and $\frac{\sigma_{\Delta V}}{\overline{\Delta V}} \leq 0.5$. The few deviant CTTs, already mentionned above, are easily identified in this plot.

We define 2 additional parameters in order to characterize the preferred brightness state of each object and the long term variations of its extreme (mimimum and maximum) light levels. The first parameter is defined as $C 1=\left\langle\frac{V_{\operatorname{med}}-V_{\min }}{\Delta V}\right\rangle$ where $V_{\text {med }}$ is the median brightness of the object in a given season, $V_{\min }$ its maximum brightness level and $\Delta V$ its photometric amplitude during this season. $C 1$ is computed for each season and then averaged over all seasons. The $C 1$ parameter thus characterizes the preferred light level of the object on the long term. Thus defined, low $C 1$ values indicate objects spending more time in the high luminosity state than in the low one. Parenago (1954) discussed a similar classification of irregular variables based on a histogram of their measured brightnesses.

The second parameter is defined as $C 2=\sigma_{V_{\min }} / \sigma_{V_{\max }}$ where $V_{\min }$ is the maximum brightness state and $V_{\max }$ the minimum one for each season. This parameter thus characterizes the long term variability (years) of the bright state relative to that of the faint state. The $C 2$ parameter can be useful to clarify the origin of the variability. For instance, in CTTs where the variability primarily results from hot spots, the minimum light level $(\simeq$ photospheric level) is expected to remain nearly constant over the years, while the maximum light level (star + hot spot) is expected to vary on the long term as the spot properties (size and/or temperature) change. Hence, CTTs with hot spots are expected to have large $C 2$ values. Conversely, CTTs whose variability is due to cold spots or circumstellar extinction will exhibit a nearly stable maximum light level ( $\simeq$ photospheric flux $)$ while the minimum light level is expected to vary as the cold spot or the occulting blob size evolve over the years. Hence, CTTs whose variability is due to cold spots and/or circumstellar extinction are expected to have low $C 2$ values.

$C 1$ and $C 2$ were computed for 35 CTTs observed over at least 7 seasons, in order to derive a reliable estimate of $\sigma_{V_{\min }} / \sigma_{V_{\max }}$. Figure 7 shows $C 1$ against $C 2$ and reveals some correlated behavior of the 2 parameters. The group of objects in the lower left part of the diagram spend most of their time close to the bright state (low $C 1$ ). They also exhibit a fairly constant maximum light level, while large variations of minimum brightness occur on the long term (see, e.g. AA Tau and GW Ori in Fig. 2a). This behavior is suggestive of non uniform circumstellar extinction occurring on the short term (weeks) with an amplitude which varies on the long term (years). In support of this hypothesis, we note that 8 of 10 UX Ori objects monitored at Mt Maidanak lie in the same region of the diagram (not shown). Also, the low frequency of such objects (5/35) agrees well with the probability of observing occultation events from the circumstellar disk as computed by Bertout (2000). Alternatively, slowly evolving cold surface spots may produce this pattern for highly inclined objects. For this group of objects, the maximum light level corresponds to the photospheric luminosity.

At the other extreme, objects in the upper right part of the diagram spend most of their time close to the faint state (high C1) and their maximum light level varies significantly on the long term (see, e.g. DF Tau and DN Tau in Fig. 2a). The variability of these objects is probably dominated by hot surface spots whose properties vary over the years. The photospheric luminosity of these objects would thus be best measured close to minimum brightness. Finally, most CTTs lie in the intermediate region of the diagram. They do not have any preferred brightness state 

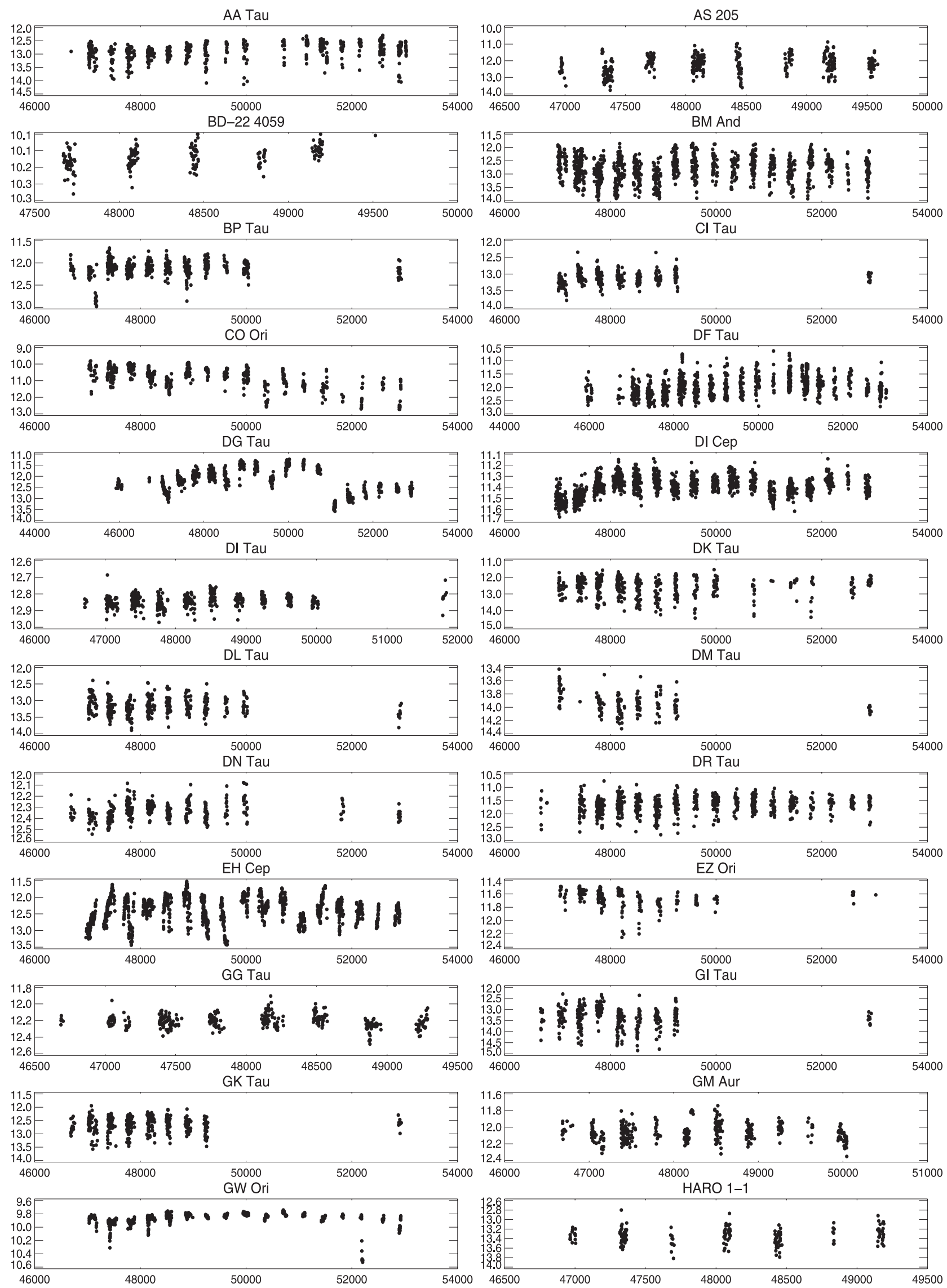

Fig. 2a. Long term $V$-band light curves of 48 CTTs observed at Mt. Maidanak. 
HARO 1-14

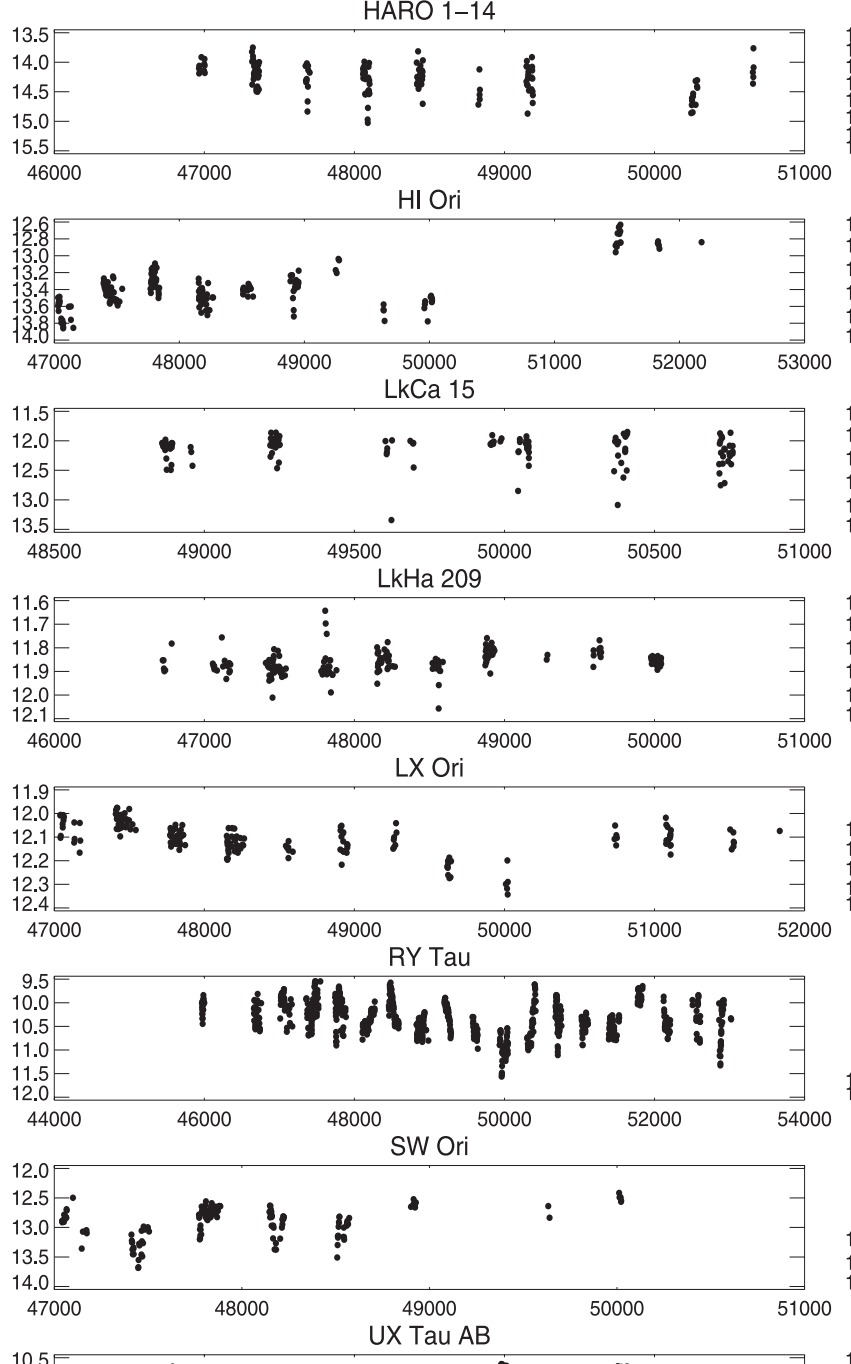

HARO 1-4
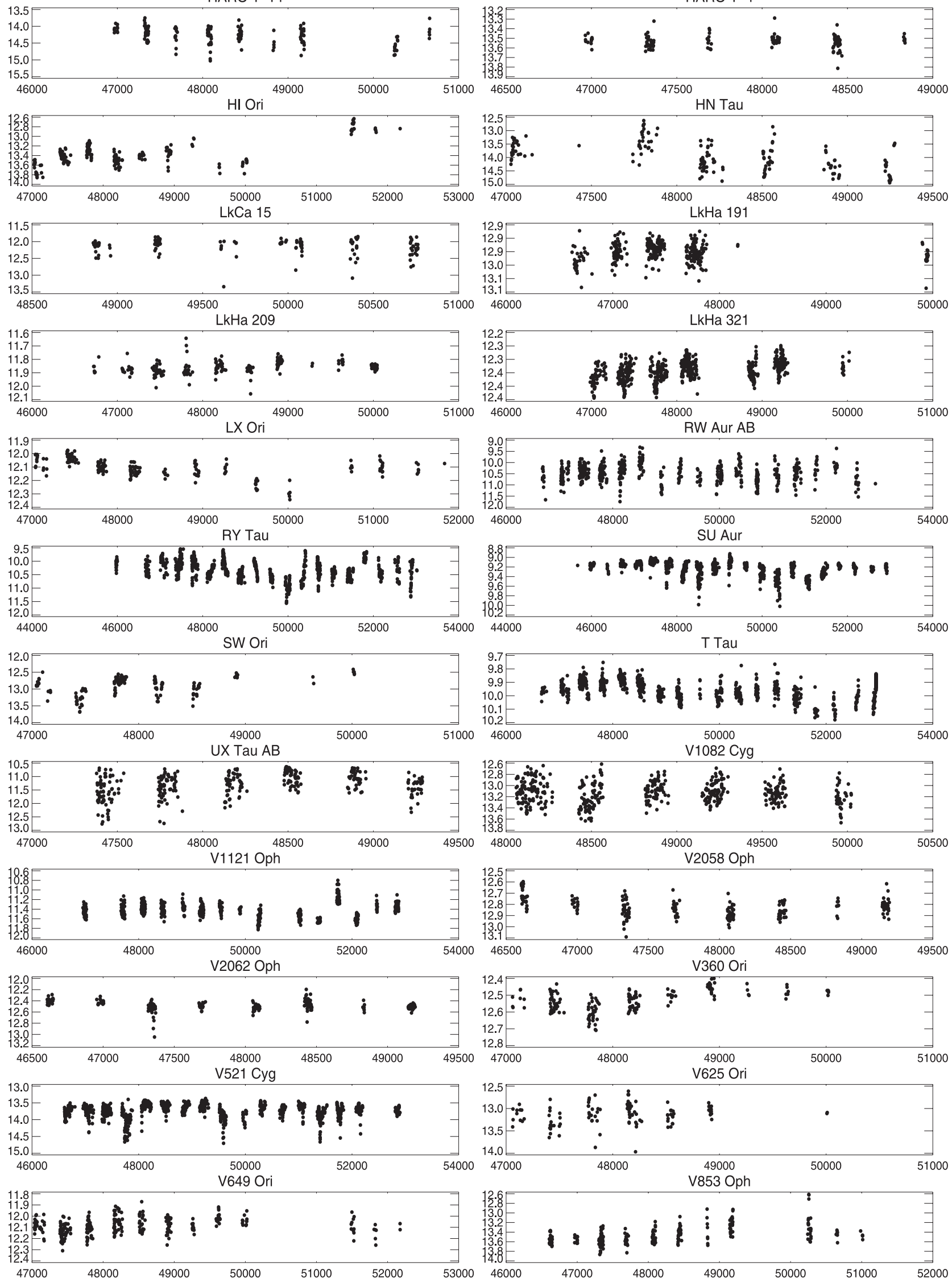

Fig. 2a. continued. 

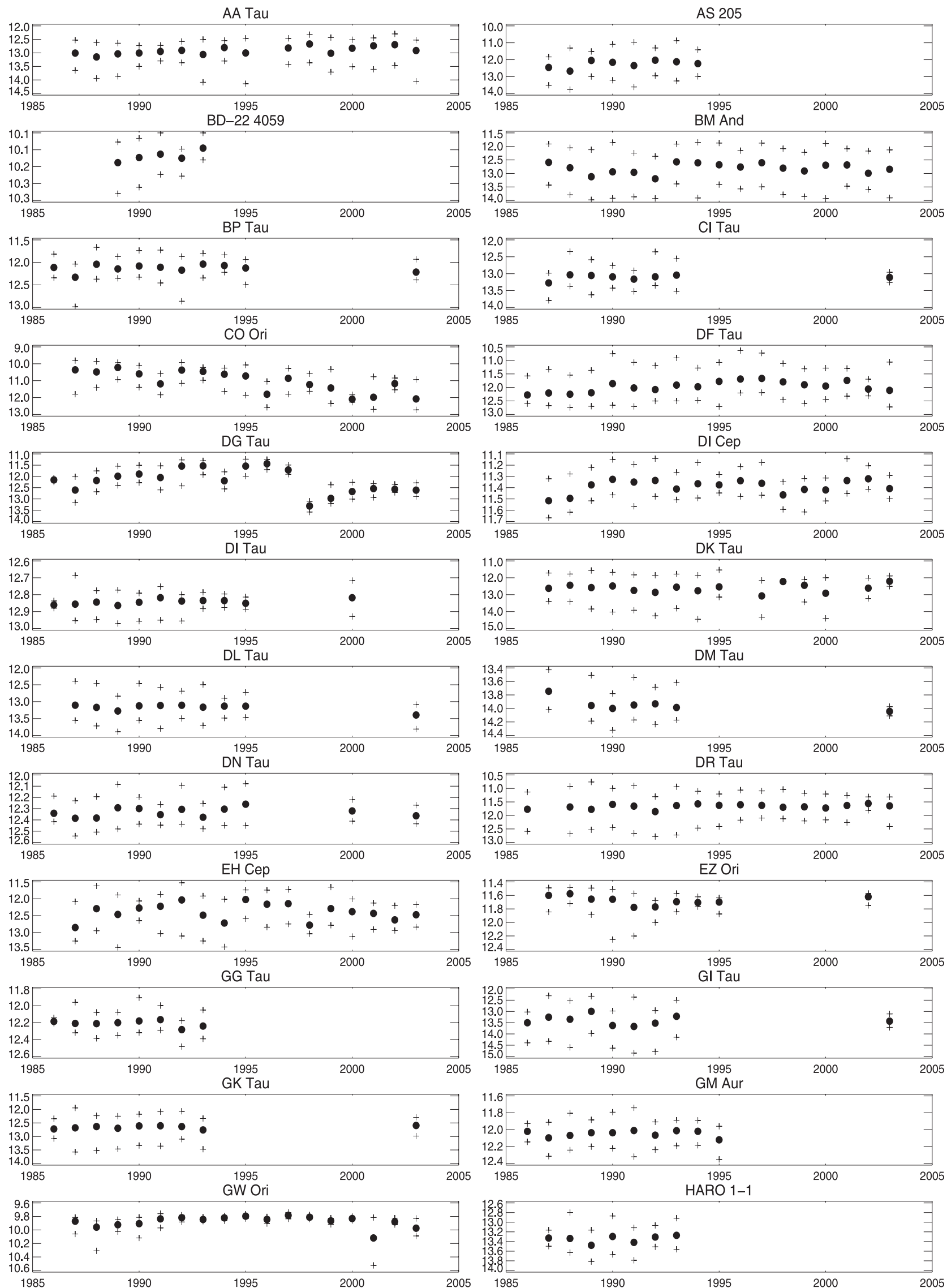

Fig. 2b. Statistical representation of the 48 CTTs light curves shown in Fig. 2a. 
HARO 1-14
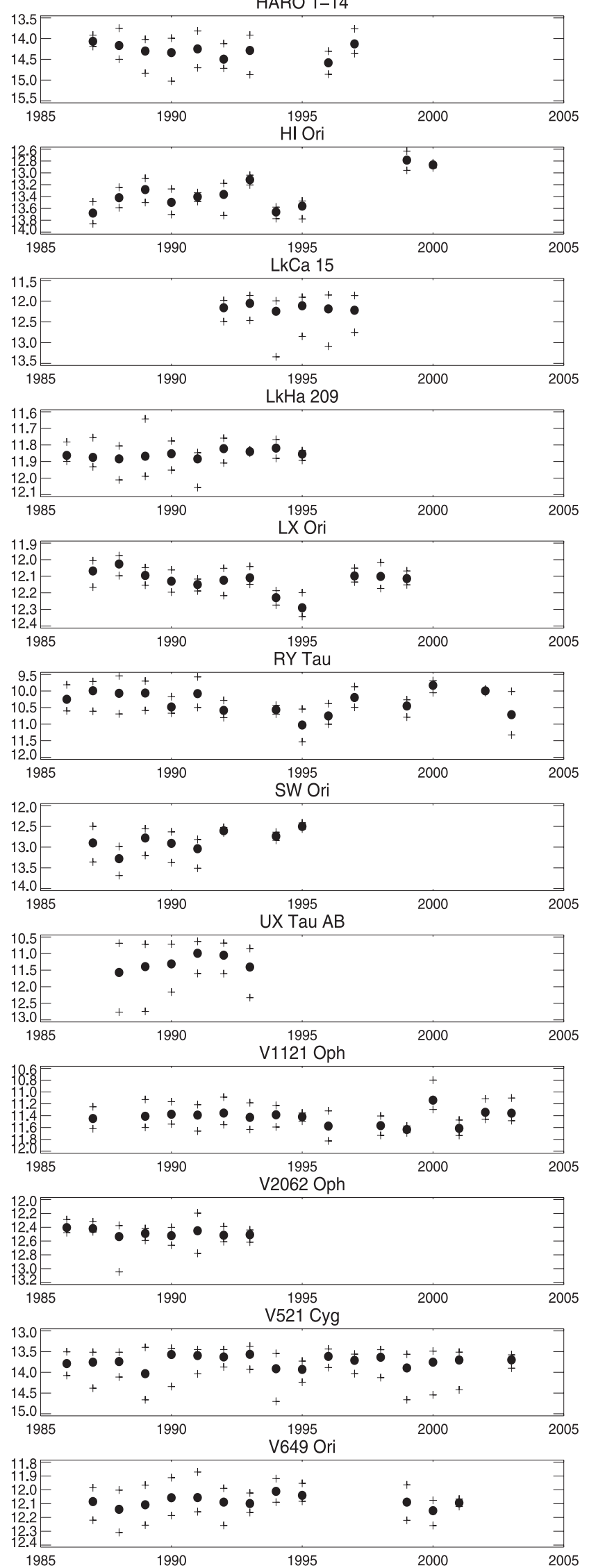
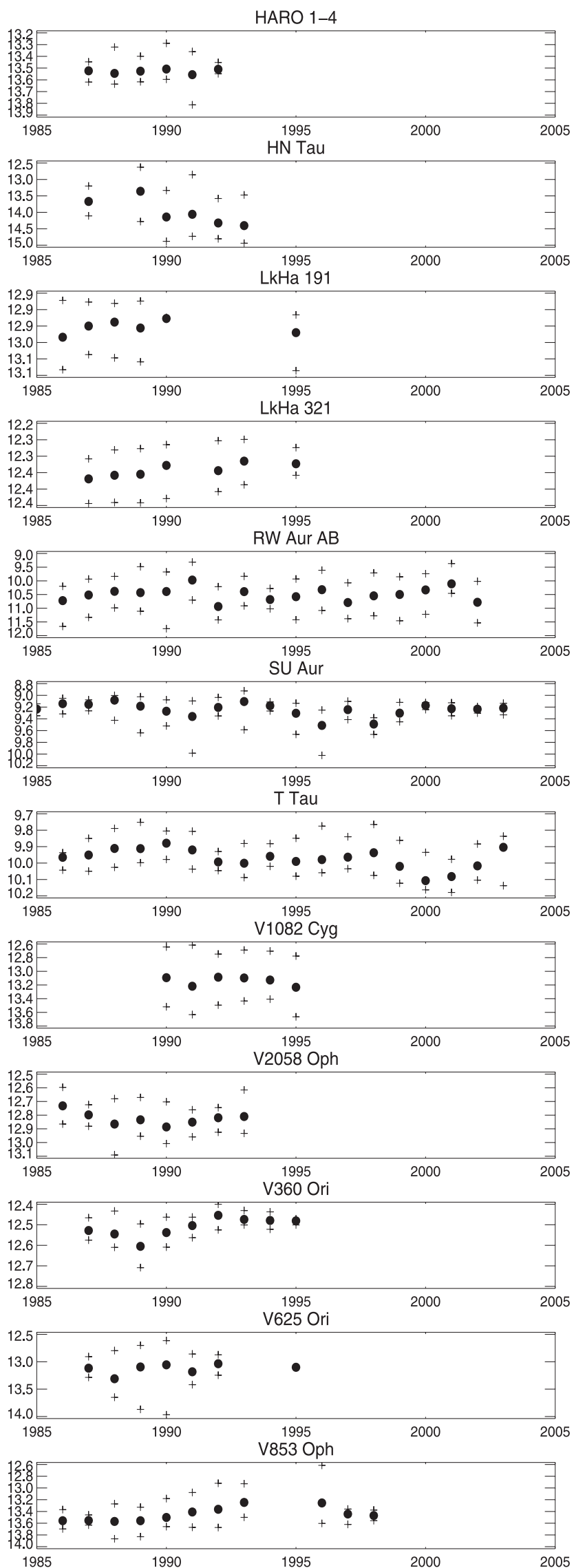

Fig. 2b. continued. 


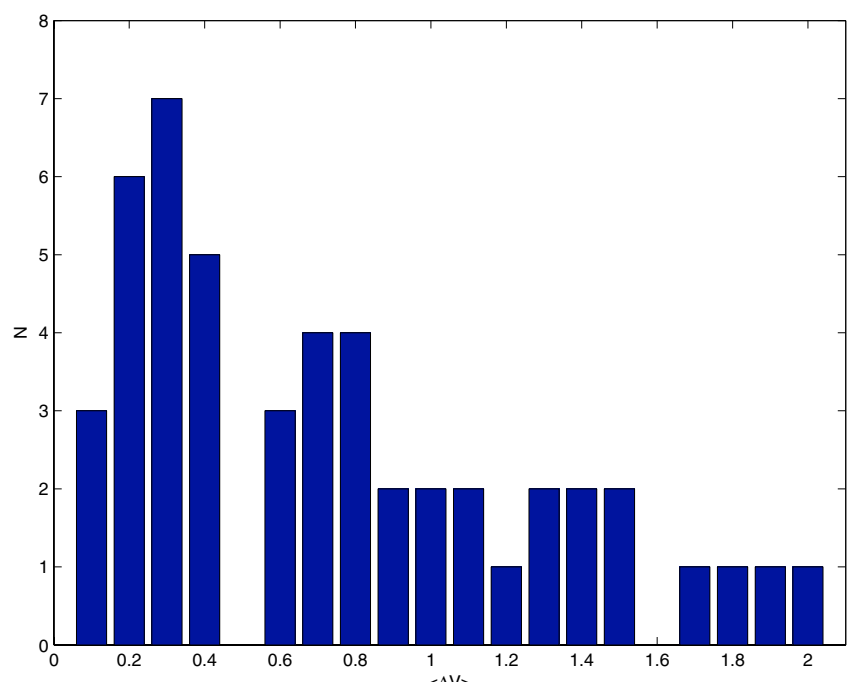

Fig. 3. Average photometric amplitude in the $V$-band for the 49 CTTs of our sample $(\overline{\Delta V})$.

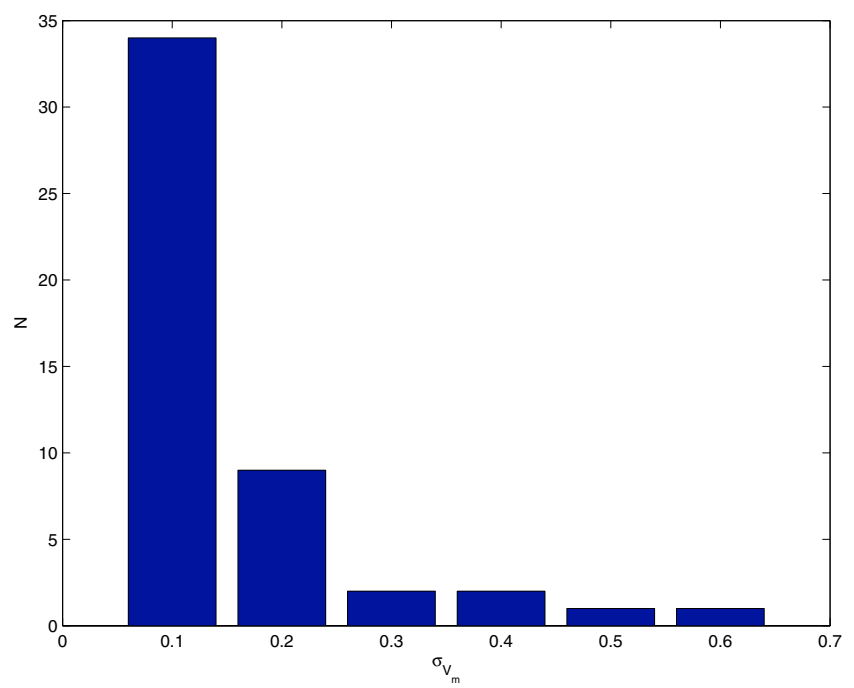

Fig. 4. Standard deviation of the seasonal mean brightness level $\left(\sigma_{V_{\mathrm{m}}}\right)$.

$(C 1 \simeq 0.5)$ and their extreme brightness levels exhibit the same amount of long term variability $(C 2 \simeq 1$ ), and sometimes (but not always) vary in a correlated fashion. The photometric variability of these objects may result from a mixture of hot and cold surface spots whose surface distribution and/or properties vary on the long term (see, e.g. DR Tau, T Tau, BM And, DI Cep in Fig. 2a). We show in an accompanying paper that WTTS, whose photometric variability is due to slowly evolving cold surface spots, lie in this intermediate region of the diagram as well.

Finally, we investigate the color behavior of the 49 CTTs in our sample. For most objects, the colors (either $(V-R)$ or $(B-V)$ ) scale linearly with brightness. Hence, the color slope can be computed from a linear least square fit. For most CTTs (39), the correlation coefficient between $V-R$ and $V$ variations is higher than 0.47 (cf. Fig. 8). Only 5 stars do not show any reliable dependence of $V-R$ on brightness (correlation factor of lower than 0.47 ) when averaged over all seasons. These stars are: GG Tau, LkH $\alpha$ 191, LkH $\alpha 321, \mathrm{LX}$ Ori, and V360 Ori. In addition, 5 CTTs demonstrate a more complex color behavior, some exhibiting a blue turnaround towards minimum brightness

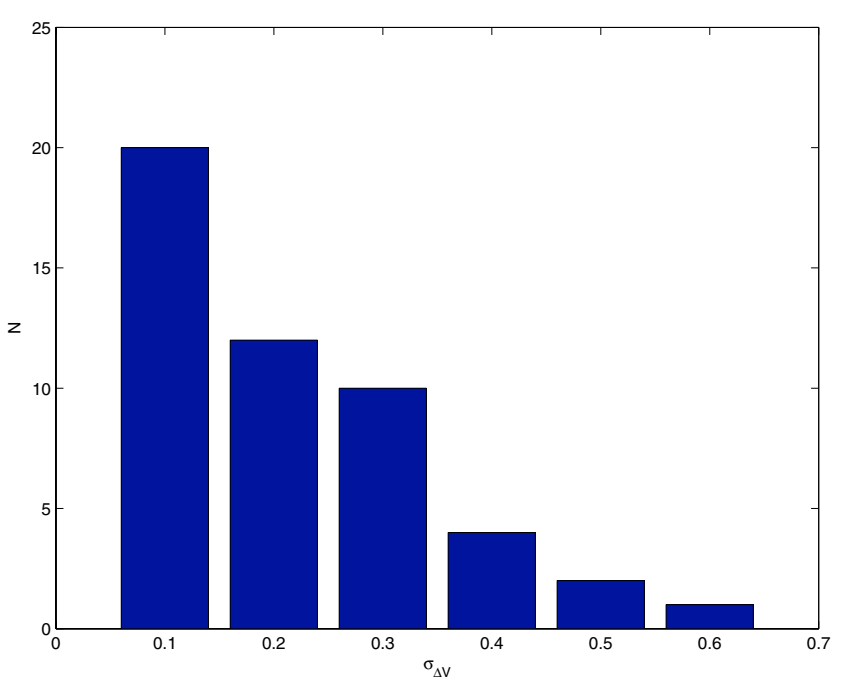

Fig. 5. Standard deviation of the seasonal photometric amplitude $\left(\sigma_{\Delta V}\right)$.

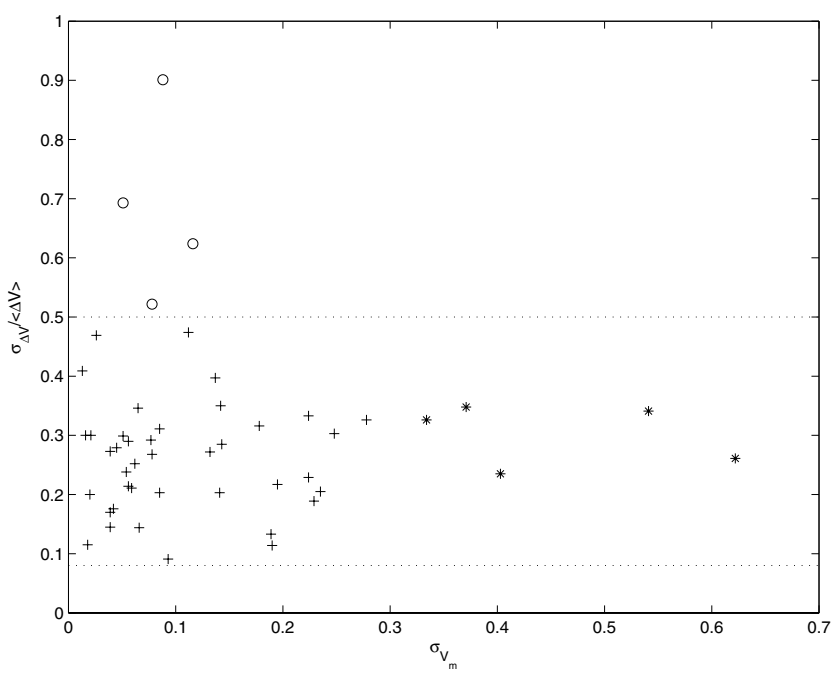

Fig. 6. The relative variation of amplitude vs. the variation of the mean brightness level. Four objects with the largest relative variations of amplitudes are shown by empty circles. Five stars with the largest changes in mean light level are denoted by asterisks.

(cf. Fig. 9). The unique color pattern of these stars (V521 Cyg, RY Tau, EH Cep, AA Tau, and CO Ori) is discussed below.

The histogram of $\frac{\Delta(V-R)}{\Delta V}$ color slopes for the 39 CTTs with linear color variations is shown in Fig. 10. The histogram peaks at values of order of $0.2-0.3$, close to the value expected for variable circumstellar extinction (0.25), assuming interstellar extinction properties. This could be taken as evidence that CTTs light variability is caused by a changing screen of circumstellar dust intercepting the line of sight to the star (e.g. Bertout 2000). However, similar color slopes can be reproduced as well by other mechanisms. We show in an accompanying paper that WTTS exhibit the same average $\frac{\Delta(V-R)}{\Delta V}$ color slope as CTTs. In WTTS, brightness and color variations result from the rotational modulation of the stellar flux by cold surface spots. Hence, color slopes usually do not uniquely distinguish between several possible sources of variability.

A similar analysis was carried out for the $\frac{\Delta(B-V)}{\Delta V}$ color slope, whose histogram is also shown in Fig. 10 and looks quite similar to the redder color, with a maximum at 0.2 . The ISM extinction 


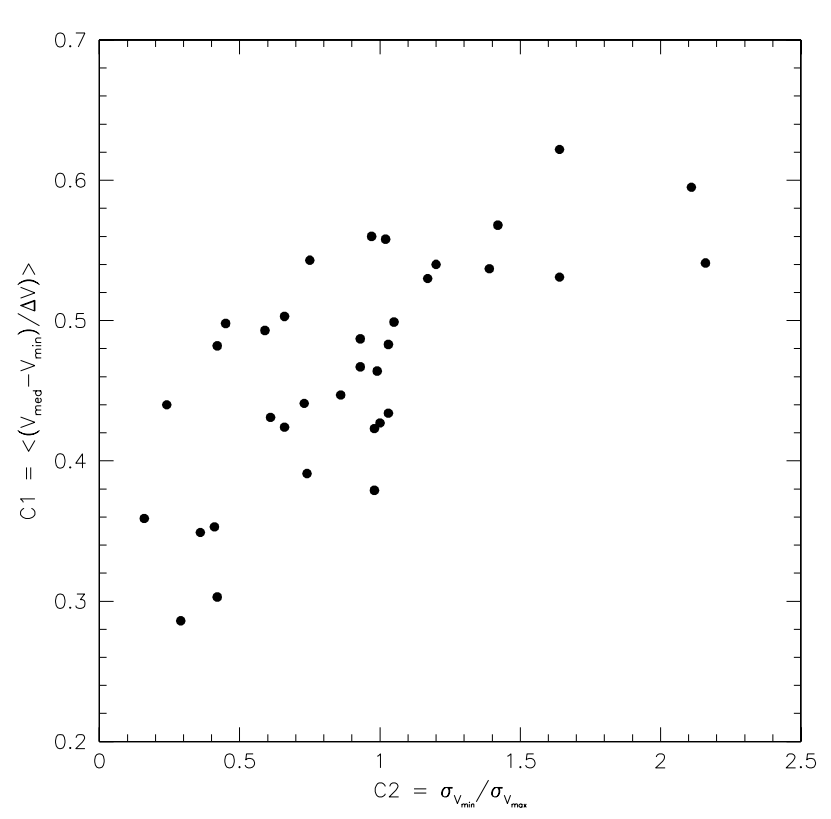

Fig. 7. The star's preferred brightness state (as measured by $C 1=$ $\left.\left\langle\frac{V_{\operatorname{med}}-V_{\min }}{\Delta V}\right\rangle\right)$ is plotted against the relative variations of its extreme brightness states (as measured by $C 2=\sigma_{V_{\min }} / \sigma_{V_{\max }}$ ). See text.
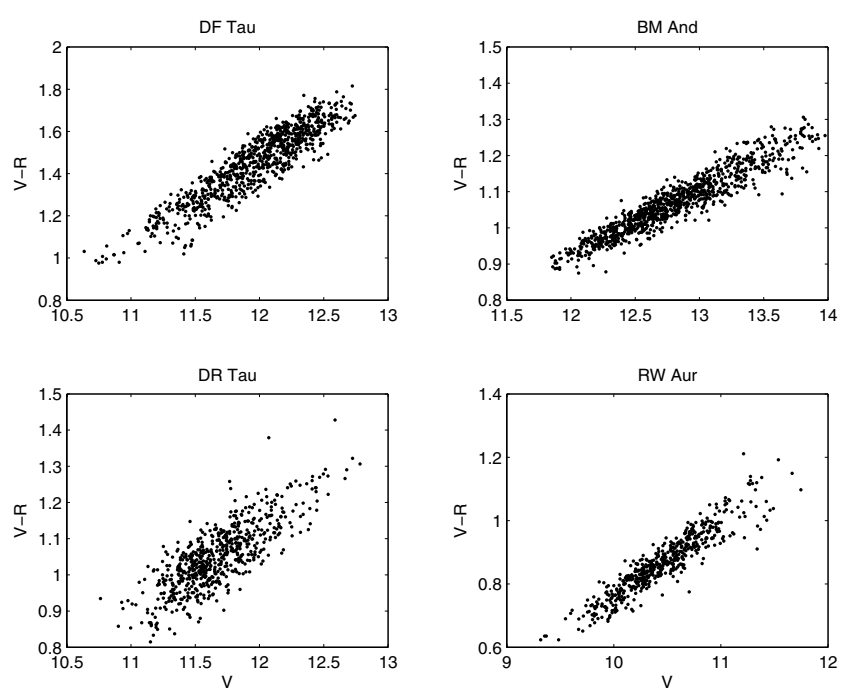

Fig. 8. Illustration of the linear relation found for most objects between the $V-R$ color and $V$ magnitude. Color slopes and correlation factors are listed in Table 2.

law for $\frac{\Delta(B-V)}{\Delta V}$ predicts a value of 0.32 if color changes are due to variable circumstellar extinction. However, besides extinction, other sources may affect the bluer colors of CTTs, for example the well known veiling phenomenon which is most important in the blue. Here again, color slopes are not sufficient to distinguish between the various possible sources of photometric variations.

\section{Discussion}

The short term (weeks) photometric variability of CTTs is usually interpreted as resulting from 3 possible causes, sometimes present simultaneously: (i) cold surface spots, presumably resulting from strong photospheric magnetic fields; (ii) hot spots at the stellar surface, related to disk accretion onto the star; and (iii) variable obscuration of the star by circumstellar matter.
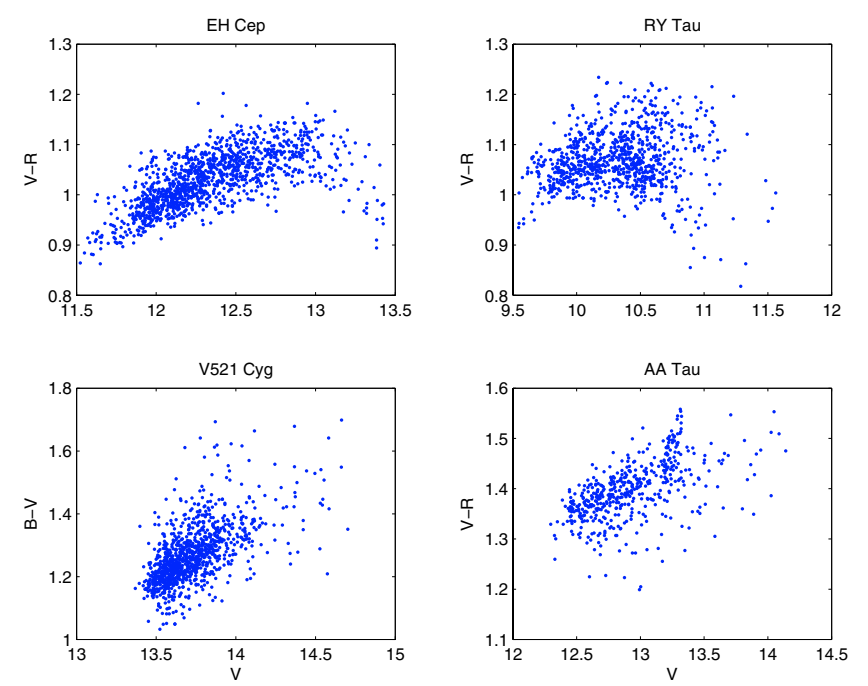

Fig. 9. Illustration of the non linear $V-R$ color variations on $V$ magnitude found for a few objects.
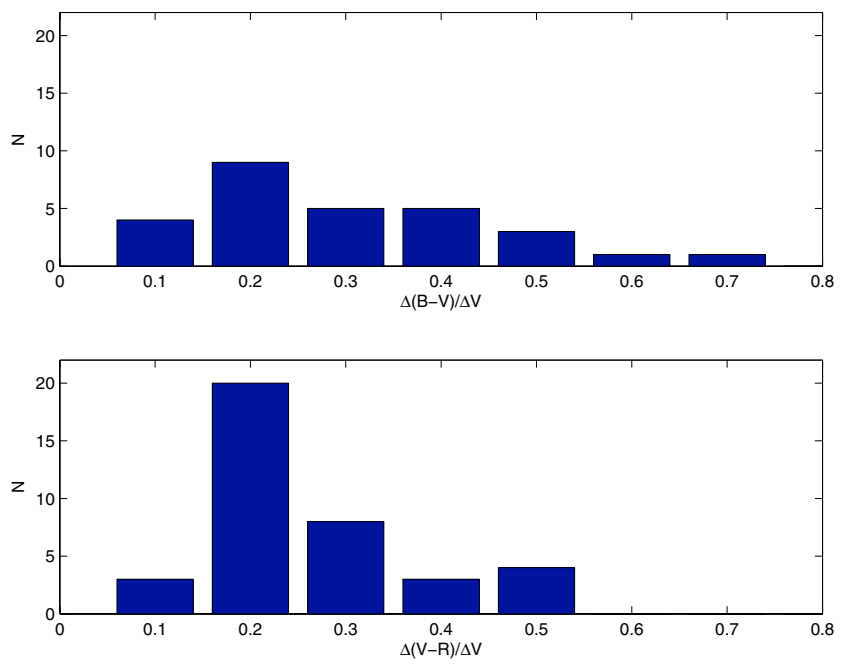

Fig. 10. Least-square color slopes. Top panel: $\frac{\Delta(B-V)}{\Delta V}$; lower panel: $\frac{\Delta(V-R)}{\Delta V}$.

The results obtained from the statistical analysis of the long term light curves of 49 CTTs are graphically summarized in the 3D plot shown in Fig. 11. From these results, we attempt to identify sub-groups of stars which appear to share the same type of long term photometric behavior and try to relate it to specific physical processes. A major difficulty is that different processes may be at work on different timescales (hours, days, weeks, years), and several sources of variability are likely to coexist. In addition, other parameters, such as the system inclination, may yield a different photometric pattern for a given source of variability. We thus merely try to identify the dominant source of variability for each subgroup, among the 49 stars with sufficient data.

The largest group of CTTs we identify in Fig. 11 consists of 37 stars which exhibit homogeneous long term photometric variability. Of these, 15 are located close to the origin of the plot exhibiting a low level of variability. These 15 stars are shown by filled squares in Fig. 11 and the other 22 stars as crosses. The spectral types of these stars span a range from G5 up to M0. This group includes, e.g., T Tau and DI Cep. CTTs in this group are characterized by relatively stable patterns of variability on the 


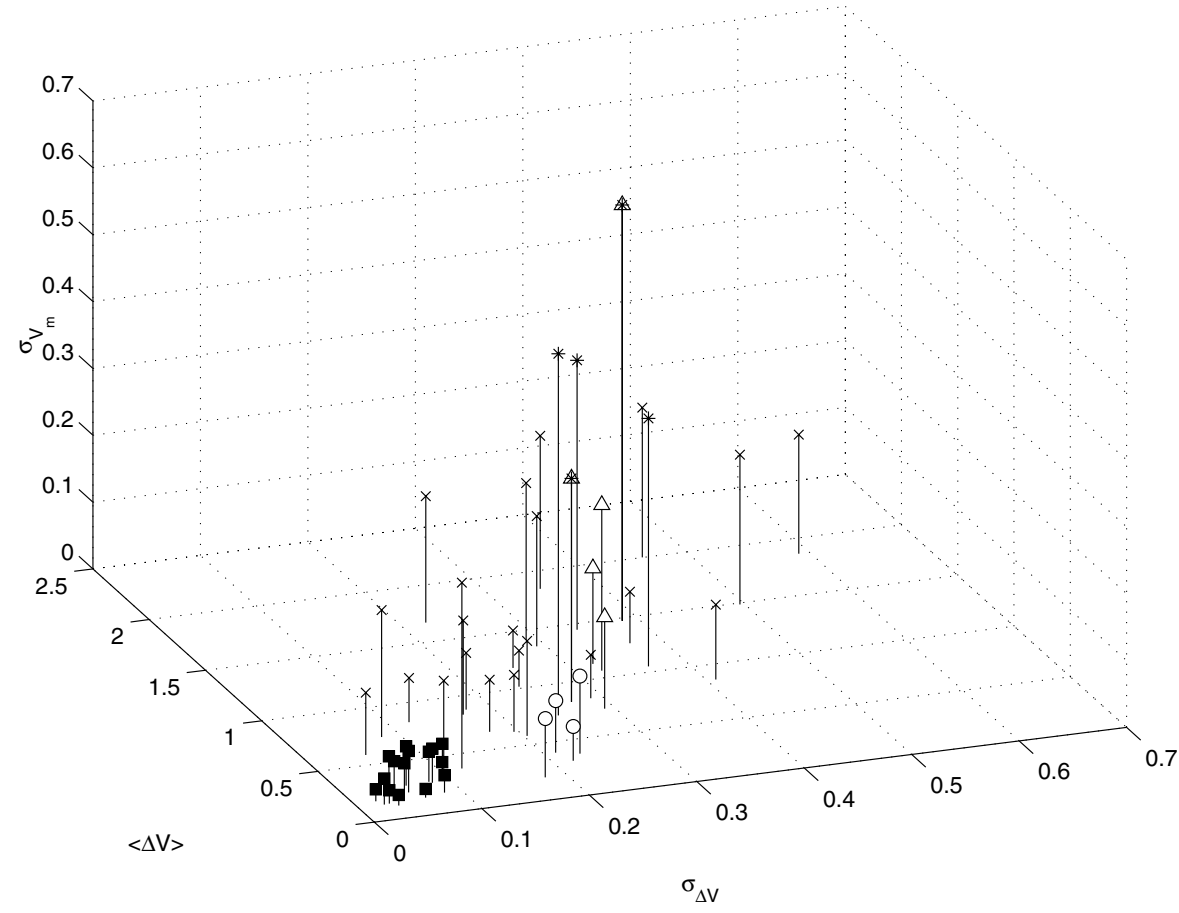

Fig. 11. A 3D plot of the statistical properties of the long term light variability of 49 CTTs. The compact group of stars located close to the origin (filled squares) exhibit the lowest degree of variability. Five stars demonstrate large changes in their mean brightness level over the years (asterisks). Another small group exhibit significant seasonal variations in their photometric amplitude (circles). Five CTTs exhibiting unusual color behavior with a blue turnaround at minimum brightness are shown as triangles.

long term. Many are known to be actively accreting from their disk and the analysis of their short term (weeks) photometric variability suggests it is dominated by hot surface spots, though cold spots as well as circumstellar extinction can also be present (Bouvier \& Bertout 1989; Vrba et al. 1993; Bouvier et al. 1995; Bertout 2000). Hence, the long term variability of these stars merely reflects variability on shorter timescales, with no significant variations from season to season.

The constancy of the light curves of these objects over the years suggests that even though the properties of surface hot spots appear to vary on the short term, possibly due to the changing accretion rate onto the star, the accretion process remains active and fairly stable on a timescale of several years. The large range of photometric amplitude displayed by CTTs in this group, from a $0^{\mathrm{m}} 1$ up to $1^{\mathrm{m}} 6$ in the $V$-band, may reflect either different accretion rates onto the star or geometric projection effects resulting from the combination of the spots latitude and the system's inclination. We do not find any correlation between photometric amplitudes and the time-averaged accretion rate in this sample using the $\mathrm{H}_{\alpha}$ flux as a proxy, which favors the latter explanation.

Besides this main group, a much smaller subgroup of deviant CTTs seen in Fig. 11 exhibits large changes in their mean brightness level over the years (DG Tau, HN Tau, UY Aur, CO Ori, and RY Tau). These stars are shown by asterisks in Fig. 11. Some of these, like UY Aur, display a smooth change of mean brightness over the years (Fig. 1). Others however, exhibit abrupt mean brightness variations from one season to the next (e.g. from 11. 71 to 13 m. 31 between 1997 and 1998 for DG Tau). The origin of these variations cannot be unambiguously assigned to a single mechanism. Large scale variations of the accretion rate onto the star could produce such a behavior. Alternatively, a variation of the circumstellar extinction on the line of sight on a timescale of years cannot be excluded. Such a timescale would then point to an obscuring screen located in the outer disk regions.

Another small group exhibits significant seasonal variations in their photometric amplitude (EZ Ori, SU Aur, V2062 Oph, and GW Ori). We have shown these stars as circles in Fig. 11. The long term light curve of these objects is characterized by a nearly constant maximum brightness level with a usually small amplitude of variability, but interrupted at times by deep fading episodes. The amplitude changes from season to season can amount to $52-90 \%$ of the average amplitude in these objects, while it amounts to only $10-47 \%$ in the rest of the CTTs sample. Some of these objects might be eclipsing binaries (e.g. GW Ori). Others may undergo partial occultation by circumstellar disk material on a timescale ranging from weeks to years (e.g. SU Aur, cf. DeWarf et al. 2003; Bertout 2000).

Finally, a last group of a few CTTs exhibit unusual color behavior with a blue turnaround at minimum brightness (V521 Cyg, RY Tau, EH Cep, AA Tau, and CO Ori). These stars are shown as triangles in Fig. 11. This likely points to variable circumstellar extinction: as the stellar photosphere is partly occulted by circumstellar material, the ratio of scattered light to direct light increases, and the system turns bluer. These stars tend to be among the earlier type stars in our sample, with spectral types from F8 to $\mathrm{K} 2$, except for AA Tau, quite a typical CTTs with a spectral type K7 (Bouvier et al. 1999, 2003, 2006). Such occultation effects are expected to be observed in only a small fraction of CTTs seen at high inclination (Bertout 2000). Two stars of this group (RY Tau and CO Ori) also belong to the small group of CTTs with large changes in their mean brightness level over the years. They are shown by two superimposed symbols (asterisks and triangles) in Fig. 11.

Our 20-year long photometric database thus provides the first opportunity to investigate the long term variability of a significant sample of CTTs. Yet, this baseline remains short compared 
to the accretion (viscous) timescale in an accretion disk, not to mention the Kelvin-Helmoltz PMS evolution timescale. Hence, while the longest photometric database available today on PMS stars, it may still miss some sources of variability acting on even longer timescales. As an illustration, the 40-yr long light curve of T Tau investigated by Mel'nikov \& Grankin (2005) exhibits a gradual rise of the mean light level from $V \simeq 10.5$ to $V \simeq 9.9$ over a timescale of about 20 years prior to 1980 .

\section{Conclusion}

The photometric variations of a large sample of CTTs have been monitored at Mt. Maidanak Observatory. More than $21000 U B V R$ photometric measurements have been collected in a uniform manner for 72 CTTs over as many as twenty years. Based on this unique database, we investigated here the long term photometric properties of a subsample of 49 CTTs that were observed at least 12 times in each of 5 or more seasons. By defining a set of statistical parameters, we characterized the long term photometric patterns of this sample of CTTs.

Several types of long term photometric patterns were identified. The most common type, shared by about $75 \%$ of the CTTs in our sample, is characterized by low to moderate amplitudes of variability and relatively stable light variations on a timescale of several years. We argue that the source of variability in these objects is likely dominated by a mixture of hot and cold surface spots. In this subgroup, 15 stars were previously investigated by Herbst et al. (1994) and classified as Type II variables. Even though the properties of the spots may change on the short term (weeks), the overall stability of the light variations over the years suggest that the underlying physical processes, i.e., accretion onto the star for hot spots and strong magnetic fields for cold spots, remain instrumental and undergo only marginal changes on the longer term.

In addition to this common pattern, a few more peculiar types of long term photometric variations are found. They are characterized by significant changes in the mean brightness level and/or photometric amplitudes over the years. We suggest that, in most cases, this type of unusual variability is dominated by variable circumstellar extinction, presumably due to the inner (short term) or outer (long term) disk regions partially occulting the central star. Occultation effects are likely to happen only in objects seen at high inclination, which explains why these patterns are more rarely observed (cf. Bertout 2000), being exhibited by only 12 CTTs over 49 in our sample. Most of the stars in this subgroup correspond to Type III variability as defined by Herbst et al. (1994). As previously noted by these authors, we find that most $(8 / 12)$ of the CTTs exhibiting this peculiar type of variability have a spectral type $\mathrm{K} 2$ or earlier, while most stars in our sample have later spectral types. Hence, this type of variability seems to preferentially (but not exclusively) affect early-type CTTs and may be related to the UXor variability pattern seen in a minority of the more massive Herbig Ae-Be stars.

Finally, we caution that photometric variability in CTTs, be it short or long term, often results from several processes acting simultaneously and which may be difficult to identify from photometry alone. Spectroscopic and polarimetric measurements obtained simultaneously with photometric ones, which have been gathered so far for very few CTTs, usually offer a clearer view of the physical processes responsible for the observed variability.

The results reported here on the long term photometric behavior of CTTs will be contrasted with a similar analysis of the long term photometric variations of WTTs from the same database in an accompanying paper.

Acknowledgements. The authors wish to thank M. Ibrahimov, S. Yakubov, O. Ezhkova, V. Kondratiev and many other observers and students, who worked or are still working in the Tashkent Astronomical Institute, for their participation in the photometric observations. We are particularly grateful to C. Dougados, C. Bertout, \& F. Ménard for their comments and suggestions pertaining to this work. We thank an anonymous referee for comments that helped to improve the manuscript. Support of the American Astronomical Society, European Southern Observatory (grant A-02-048), and International Science Foundation by Soros (grant MZA000) and a CRDF grant (ZP1-341) is acknowledged. This work was also supported by a NATO Collaborative Linkage grant for European countries (PST.CLG.976194) and by an ECO-NET program of the French Ministry of Foreign Affairs, which we both gratefully acknowledge. W.H. gratefully acknowledges the support of NASA through its Origins of Solar Systems Program.

\section{References}

Bertout, C. 2000, A\&A, 363, 984

Bouvier, J., \& Bertout, C. 1989, A\&A, 211, 99

Bouvier, J., Covino, E., Kovo, O., et al. 1995, A\&A, 299, 89

Bouvier, J., Chelli, A., Allain, S., et al. 1999, A\&A, 349, 619

Bouvier, J., Grankin, K. N., Alencar, S. H. P., et al. 2003, A\&A, 409, 169

Bouvier, J., Alencar, S. H. P., Boutelier, T., et al. 2006, A\&A, in press

DeWarf, L. E., Sepinsky, J. F., Guinan, E. F., Ribas, I., \& Nadalin, I. 2003, ApJ, 590,357

Ehgamberdiev, S. A., Baijumanov, A. K., Ilyasov, S. P., et al. 2000, A\&AS, 145, 293

Grankin, K. N. 1997, Astron. Lett., 23, 700

Grankin, K. N. 1998, Astron. Lett., 24, 580

Grankin, K. N. 1999, Astron. Lett., 25, 611

Grankin, K. N., Ibragimov, M. A., Kondratiev, V. B., Melnikov, S., \& Shevchenko, V. 1995, AZh, 72, 894

Herbig, G. H., \& Bell, K. R. 1988, Lick Obs. Bull., 1111, 1

Herbst, W., Herbst, D. K., Grossman, E. J., \& Weinstein, D. 1994, AJ, 108, 1906 Herbst, W., \& Shevchenko, V. S. 1999, AJ, 118, 1043

Joy, A. H. 1942, PASP, 54, 15

Joy, A. H. 1945, ApJ, 102, 168

Landolt, A. U. 1983, AJ, 88, 439

Landolt, A. U. 1992, AJ, 104, 340

Mel'nikov, S. Y., \& Grankin, K. N. 2005, Astron. Lett., 31, 109

Menard, F., \& Bertout, C. 1999, in The Origin of Stars and Planetary Systems, ed. C. J. Lada, \& N. D. Kylafis (Kluwer Academic Publishers), 341

Nikonov, V. 1976, Izvestiya KrAO, 54, 3

Parenago, P. 1954, Publ. Sternberg Astron. Inst., 25

Shevchenko, V. S. 1989, Herbig Ae/Be Stars (in Russian) (Tashkent: FAN)

Shevchenko, V. S., Grankin, K. N., Ibragimov, M. A., Melnikov, S. Y., \& Yakubov, S. D. 1993a, Ap\&SS, 202, 121

Shevchenko, V. S., Vitrichenko, E. A., Grankin, K. N., Ibragimov, M. A., \& Melnikov, S. Y. 1993b, SvA Lett., 19, 125

Shevchenko, V. S., Grankin, K. N., Melnikov, S. Y., \& Lamzin, S. A. 1998, Astron. Lett., 24, 528

Strauzis, V. 1977, Multicolor Stellar Photometry (Vilnius: Mokslas)

Vrba, F. J., Chugainov, P. F., Weaver, W. B., \& Stauffer, J. S. 1993, AJ, 106, 1608 